\title{
Astym therapy improves function and range of motion following mastectomy
}

\author{
This article was published in the following Dove Press journal: \\ Breast Cancer:Targets and Therapy \\ 8 March 2016 \\ Number of times this article has been viewed
}

\author{
Claire C Davies' \\ Dorothy Brockopp ${ }^{2}$ \\ Krista Moe ${ }^{2}$ \\ 'Baptist Health Rehabilitation, \\ ${ }^{2}$ Research Department, Baptist Health \\ Lexington, Lexington, KY, USA
}

Correspondence: Claire C Davies Baptist Health Rehabilitation, Baptist Health Lexington,

1800 Nicholasville Road, Lexington, KY 40503, USA

Tel + I 8592606673

Fax + I 859260643 |

Email claire.davies@bhsi.com

\begin{abstract}
Hypersensitive scar tissue formation along the incision line and drain site is a common side effect following mastectomy. If this scar tissue is not addressed, it can lead to decreased flexibility and function in the involved upper quadrant. Astym ${ }^{\circledR}$ treatment is a new approach to soft tissue injuries, and is evidenced in animal studies to promote the healing and regeneration of soft tissues. It has also been found to reduce pain and increase function in people with soft tissue dysfunction. The aim of Astym treatment is to engage the regenerative mechanisms of the body in order to resorb scar tissue, stimulate tissue turnover, and regenerate soft tissues. Handheld instrumentation is applied topically to locate and treat the underlying dysfunctional soft tissue through specific protocols for the application of particular pressures and shear forces. The purpose of this study was to examine the effects of Astym treatment on activities of daily living in women who had undergone a mastectomy following a diagnosis of breast cancer. A quasi-experiment involving 40 women, following a mastectomy, evaluated five outcome measures pre- and post-Astym treatment. All five measurement scores: Disabilities of the Arm, Shoulder, and Hand Outcome Measure; a clothing questionnaire on their ability to wear a bra; Patient-Specific Functional Scale; active range of motion of shoulder flexion; and active range of motion of abduction were also measured and all demonstrated significant changes. In this study, Astym treatment improved active range of motion in the involved quadrant and also improved function in patients following a mastectomy.
\end{abstract}

Keywords: breast cancer, quality of life, scar tissue treatment, physical therapy

\section{Introduction}

It is estimated that there are 2.9 million breast cancer survivors (BCSs) in the USA, ${ }^{1}$ who have undergone common treatments such as surgery and/or radiation. ${ }^{2}$ Following this type of treatment, scar tissue can develop along the incision and around the drain sites of the involved upper quadrant. If a woman has undergone radiation therapy, adhesions and soft tissue fibrosis can form further adding to scar tissue. ${ }^{2}$ The scar tissue can tighten and become restrictive leading to decreased function and flexibility. ${ }^{3}$ Over time, these common complications advance to activity limitations and participation restrictions. ${ }^{3,4}$

Scar tissue often goes untreated and becomes painful and hypersensitive. Restrictions in tissue flexibility reduce movement at the shoulder affecting function. Studies have demonstrated that up to $86 \%$ of BCSs have a shoulder limitation following complete axillary dissection and $45 \%$ have limitation after a sentinel node biopsy. ${ }^{3,5}$ Currently, increasing range of motion through exercise and stretching is a main focus of 
physical therapy (PT), but the scar tissue adhesions routinely interfere with progress and optimal outcomes. PT, including manual therapy, early postoperatively can help reduce swelling, pain, and associated scar formation. ${ }^{3}$

Astym ${ }^{\circledR}$ therapy (Performance Dynamics, Inc., Muncie, IN, USA) is a relatively new, noninvasive, conservative therapeutic approach that addresses soft tissue dysfunction by relying in part on the use of cellular mediators and growth factors to assist in the healing and regeneration of tissues. ${ }^{6}$ It is evidenced in animal studies to engage the regenerative mechanisms of the body and promote healing of tissues. ${ }^{78}$ It has also been found to reduce pain and increase function in people with soft tissue dysfunction. ${ }^{6,9-11}$ The aim of Astym treatment is to engage the regenerative mechanisms of the body in order to resorb scar tissue, stimulate tissue turnover, and regenerate soft tissues. ${ }^{6}$ Handheld instrumentation is applied topically to locate underlying dysfunctional soft tissue and then transfer particular pressures and shear forces to the dysfunctional tissue through specific protocols and patterns developed from scientific and clinical study. ${ }^{6}$ The Astym instrumentation is designed to assess the presence of dysfunctional tissue by amplifying the tactile sensation of the underlying texture of the soft tissues in order to provide the treating clinician with indications where rough or improperly organized tissue is located. Once an area of potential dysfunctional tissue is located, the clinician applies appropriate pressures and shear forces to that tissue, aimed at initiating a reparative cellular response in dysfunctional tissue. ${ }^{6}$ In vivo studies revealed that the Astym protocols improved tissue repair, increased limb function, and normalized movement patterns in an animal model. ${ }^{7,8}$ Further, Astym treatment resulted in a significant increase in both fibroblast activation and fibroblast number, as well as the production of fibronectin, which together with interstitial collagens may interact to form a fibrillar component of the extracellular matrix. ${ }^{7,8}$ The increase in fibronectin is notable in that fibronectin is thought to be required for normal collagen organization and deposition by fibroblasts. This increase in fibronectin has the potential to guide cell and tissue behavior during healing as a function of its unique mechanical and bioactive properties. ${ }^{12,13}$

Astym therapy has been shown to increase movement and decrease pain in patients with inappropriate fibrosis and in patients with other soft tissue dysfunctions. ${ }^{6,-11}$

Davies and Brockopp, ${ }^{14}$ in a previous pilot study, demonstrated that Astym therapy increased active range of motion (AROM) of shoulder flexion and abduction, and improved Disabilities of the Arm, Shoulder, and Hand Outcome
Measure (DASH) scores in patients with scar tissue around their mastectomy incision, drain site, and axilla. ${ }^{14}$ The same study also reported that $56 \%$ of women were unable to wear a bra before Astym treatment, suggesting hypersensitivity of scars and clothing restrictions that affect BCSs. In this study, after Astym treatment, all women could wear a bra, without issue, indicating that Astym therapy has a normalizing, desensitizing effect on touch and pressure in the involved tissue. ${ }^{14}$

It is important to note that Astym treatment is different in goals and application from the Instrument-Assisted Soft Tissue Mobilization (IASTM) techniques, such as Graston Technique $^{\circledR}$. IASTM uses tooled cross-friction massage to mechanically break apart tissue, ${ }^{15,16}$ whereas Astym therapy engages the regenerative mechanisms of the body aiming to repair damaged tissue and resorb scar tissue. ${ }^{6-8}$ IASTM's role in the treatment of soft tissue dysfunction is unclear, as IASTM has been shown to 1) not improve treatment results for ankle injury, ${ }^{17}$ 2) be comparable to no treatment being provided in tendinopathy, ${ }^{18}$ and 3) result in a significant increase in pain and a significant decrease in the perception of function, where the ability to perform activities of daily living (ADL) decreased following IASTM. ${ }^{19}$

The purpose of this study was to examine the effect of Astym treatment on ADL of women who had undergone a mastectomy following a diagnosis of breast cancer. The specific aims of the study were to examine differences in 1) a patient's subjective assessment of functional disability through the DASH score, 2) the ability to wear a bra through a clothing questionnaire, 3 ) up to five functional activities women have reported to be difficult after mastectomy using the Patient-Specific Functional Scale (PSFS), 4) shoulder flexion, and 5) shoulder abduction, before and after a course of Astym therapy.

\section{Participants and methods}

A convenience sample of patients referred to the PT clinic with complaints of decreased flexibility, chest pain, or tight scar tissue following breast cancer treatment, which included surgery and radiation, were identified and asked if they would like to participate in the study. Participants were women over the age of 18 years, who had undergone a unilateral or bilateral mastectomy for breast cancer, with or without axillary dissection, and were referred for PT at a minimum of 3 weeks post-surgery. Three weeks allowed the skin time to heal post-mastectomy. Exclusion criteria consisted of being non-English speaking and having lymphedema $>$ stage 1 , acute infection at the treatment site, open wound at baseline, active metastatic site, acute deep 
vein thrombosis, hemophilia or other clotting disorders, autoimmune dysfunctions, fibromyalgia, diabetes, obesity (bruising) and petechiae, pharmacology concerns to the skin, lumpectomies, keloid scarring, immediate breast reconstruction with spacers, previous shoulder surgery, and fractures or injury that would interfere with baseline measures. Once the participants agreed, met the inclusion criteria, and informed consent was obtained, they completed the following outcome measures: the DASH, the clothing questionnaire, and the PSFS.

\section{Outcome measures}

All outcome assessments and AROM measurements were collected at initial evaluation and discharge. The DASH questionnaire measures upper extremity function. ${ }^{20}$ The DASH is a 30 -item self-report questionnaire that includes items relating to social functioning (eg, occupation and family), physical functioning (eg, home management, recreational activities, and ADLs/self-care), and psychological function (self-image). ${ }^{21,22}$ Range of scores possible is 0\%-100\% where 0 means no disability and $100 \%$ means severe disability. ${ }^{5,21}$ DASH items are scored from 1 to 5 with 1 being no difficulty and 5 severe difficulty. Scoring of the DASH was according to the instructions (total score = [sum of $n$ responses] $/[n-1] \times 25)$, where $n$ is the number of completed responses. ${ }^{5,22}$ Swisher et $\mathrm{al}^{21}$ stated that the DASH is an appropriate outcome measure for patients with breast cancer due to the many musculoskeletal issues in the upper extremity an individual may have.

The clothing questionnaire was designed by the investigator and tested in a pilot study ${ }^{14}$ (Table S1). The investigator included a question sheet for the subjects to complete as part of the initial paperwork. This sheet contained questions regarding the woman's ability to wear a bra or not and other questions related to body image.

The functional activities that caused women difficulty were measured by the PSFS. ${ }^{23}$ This questionnaire was originally designed for the orthopedic population and can be used to quantify activity limitation and measure patient functional outcomes. ${ }^{23}$ Participants were asked to list up to five difficult functional activities to help identify the specific activities these women found challenging. Collecting the PSFS information at initial evaluation and at discharge helped determine appropriate use of the PSFS in this population.

Shoulder flexion and abduction AROM were measured, in degrees, by the same physical therapist as these movements are often the most limited following mastectomy., ${ }^{5,27}$ Measurements were taken in supine position using a goniometer.
Universal goniometry has reliability of intraclass correlation coefficient ( $95 \%$ confidence interval [CI]): flexion $(0.69,95 \%$ CI $0.40-0.91)$ and abduction $(0.69,95 \%$ CI $0.37-0.92) .{ }^{28}$

\section{Intervention}

The Astym therapy protocol used in this study was previously used in the pilot study by Davies and Brockopp. ${ }^{14}$ The same therapist performed Astym treatment on all participants in the study. Manual lymphatic drainage (MLD) was used in the treatment proximally to open up the appropriate lymph nodes to encourage lymph flow prophylactically to prevent infection and educate the women on touching themselves to develop their new body image. Women are afraid to touch their chests following surgery and/or radiation, and using MLD allows women to touch what is often a numb area as well as become comfortable with their new shape. Astym therapy protocol followed with pressure adjusted for the participant's comfort. The Astym instruments are used with gentle gliding strokes across the pectoral muscle to the axilla followed by specific strokes to address the mastectomy incision adhesions and the axillary scar, if present. Figure S1 shows a photograph of one of the tools used for the technique. Further strokes around the drain site can also be used. Treatment direction assists the lymph fluid flow to regional lymph nodes, and strokes were adjusted to the patient's tolerance. MLD followed the Astym therapy as appropriate.

Astym therapy always includes a component of therapeutic loading. In this study, the specific therapeutic exercises included active assisted range of motion (AAROM) or AROM flexion and abduction in supine position, blade squeezes in standing or sitting position, pectorals corner stretches, and postural reeducation exercises, and stretching into flexion and abduction will be implemented in supine and standing position to stay consistent. Therapeutic exercises started with AAROM and were progressed as each participant tolerated. Home instructions, including stretching exercises and activity guidelines, were given to each participant.

Treatment was administered twice a week with at least 2 days between treatments to allow regenerative mechanisms to take effect and scar tissue time to resorb. This treatment was repeated until up to eight treatments had occurred over a 4-6-week period.

\section{Ethics}

The study was reviewed by the Institutional Review Board at Baptist Health Lexington, Lexington, KY, USA. All participants gave their written informed consent to participate in this study. 


\section{Statistical analysis}

Both inferential and descriptive statistics were used to analyze the data. Descriptive statistics described the sample, and inferential statistics were used to look at differences in all variables before and after the intervention (Astym treatment). Five variables were examined for change in the participants following Astym treatment: DASH score, PSFS, clothing questionnaire, and change in flexion and abduction AROM. Given that dependent $t$-test was used and five variables were examined, a Bonferroni adjustment was made resulting in an $\alpha$ level of 0.01 significance $(0.05 / 5=0.01)$. The statistical evaluation was completed using SPSS for Windows version 21.0 (SPSS Inc, Chicago, IL, USA).

\section{Results}

\section{Participant demographics}

A total of 42 BCSs participated in this study with an age range of 33 and 75 years (mean 52.0 years, standard deviation [SD] 11.4). Two participants did not complete the DASH questionnaire posttreatment, so those data were not used in the analysis. Therefore, the data of 40 women were analyzed. The mean time of referral for PT treatment since mastectomy surgery was 7.7 months (SD 13.5). The mean numbers of Astym treatments completed during PT sessions were 6.7 (SD 1.8).

\section{Functional outcomes}

There were significant and clinical meaningful differences in functional outcomes following Astym treatment: DASH pre- and post-Astym treatment, $t=3.38$ (95\% CI 3.95-15.72; $P<0.01)$; PSFS pre- and post-Astym treatment, $t=-5.47$ (95\% CI -2.56 to $-1.12 ; P<0.01$ ); and the clothing questionnaire pre- and post-Astym treatment, $t=5.0$ (95\% CI 2.37 to $5.58 ; P<0.01)$. Mean improvements in the DASH, PSFS, and clothing questionnaire scores were 10, 2, and 4, respectively.

\section{Shoulder AROM}

There were significant differences in both flexion and abduction AROM following treatment of Astym; flexion pre- and
post-Astym treatment, $t=-5.43(95 \% \mathrm{CI}-23.96$ to -10.95 ; $P<0.01$ ); and abduction pre- and post-Astym treatment, $t=-4.45$ (95\% CI -28.18 to $-10.57 ; P<0.01)$. Mean improvement in AROM was $17^{\circ}$ flexion and $19^{\circ}$ abduction (Table 1 ). No harms were reported.

\section{Discussion}

Tight scar tissue development from treatments for breast cancer decreases shoulder mobility and limits upper quadrant function and continues to be an ongoing issue for BCSs. ${ }^{2,24,25,29}$ The results of this study demonstrate that BCSs who receive early Astym treatment have a significant and clinical meaningful improvement in shoulder abduction, forward flexion, DASH, PSFS, and clothing tolerance (ability to wear a bra). It was also noted that these women had some desensitization of the skin to allow touch as well as tolerate clothing better. These findings support a previous study by Davies and Brockopp. ${ }^{14}$ All five outcome measures taken at initial evaluation and discharge demonstrated a significant clinical change after Astym treatment.

The DASH demonstrated a change of 9.8 points in functional ability and participation in activities following Astym treatment that supports the minimal detectable clinical change of 10 points found in previous studies. ${ }^{30-32}$ Previously, Davies and Brockopp ${ }^{14}$ only used the DASH to identify functional deficit, and therefore their study lacked a detailed ability to individualize measurements to fully capture the extent of the participants' actual deficit. Using the PSFS allowed the participant to identify their specific issues with function and work toward improving those individual scores that were most important to them. There was a significant meaningful change in the PSFS score as the change of 1.84 points supports previous research of a minimal detectable overall change of 2 points. ${ }^{23}$

An additional outcome measure was the use of the clothing questionnaire. From the previous study by Davies and Brockopp, ${ }^{14}$ women identified not being able to wear a bra as an issue. Using the clothing questionnaire allowed the identification of further information on if a participant can wear a bra, if she can wear it all day, touching the scars, as

Table I Overall change in five outcome measure scores from initial evaluation to discharge and significance

\begin{tabular}{|c|c|c|c|c|c|c|}
\hline Outcome measure & Initial evaluation mean & Discharge mean & Change & $t$ & $95 \% \mathrm{Cl}$ & $P$-value significance \\
\hline DASH & 32.78 (SD 22.69) & 22.94 (SD 18.67) & 9.8 & 3.4 & 4.0 to 15.7 & $<0.01$ \\
\hline PSFS & $5.3(\mathrm{SD} 2.92)$ & 7.14 (SD 2.64) & -1.84 & -5.2 & -2.6 to -1.1 & $<0.01$ \\
\hline Clothing questionnaire & 19.38 (SD 9.16) & 15.40 (SD 7.76) & 3.98 & 5.0 & 2.4 to 5.6 & $<0.01$ \\
\hline Flexion range of motion $\left({ }^{\circ}\right)$ & I 36.45 (SD 27.20) & 153.90 (SD |4.33) & 17.45 & -5.4 & -24.0 to -11.0 & $<0.01$ \\
\hline Abduction range of motion $\left({ }^{\circ}\right)$ & I 34.80 (SD 36.02) & I54.I8 (SD 21.25) & 19.38 & -4.5 & -28.2 to -10.6 & $<0.01$ \\
\hline
\end{tabular}

Abbreviations: $\mathrm{Cl}$, confidence interval; DASH, Disabilities of the Arm, Shoulder, and Hand Outcome Measure; SD, standard deviation; PSFS, Patient-Specific Functional Scale. 
well as other body image issues. This clothing questionnaire provided very useful information to understand how the participants were dealing with their surgery and if they needed referral for counseling.

Shoulder range of motion had a clinically significant and meaningful change in both flexion $\left(17.45^{\circ}\right)$ and abduction $\left(19.38^{\circ}\right)$. This supports previous research of a minimal detectable change by one evaluator which ranged from $11^{\circ}$ to $16^{\circ} .{ }^{32}$ The change in range of motion will help improve function following surgery and enable the women to participate in activities following surgery.

Finally, a move toward normalization through desensitization seems to occur following Astym therapy as women who initially reported being unable to tolerate a bra or clothing on their skin reported that hypersensitivity to wearing a bra or clothing on the skin was resolved following treatment. Women went from not being able to touch their chest initially to then being able to feel comfortable with moving their skin. This desensitization is important in the overall well-being of the participant. Different age ranges were included in the study (range: $33-75$ years), and improvement occurred in all ages of skin to allow flexibility for functional improvement.

\section{Strengths and limitations}

A strength of this study is that this continues to be one of the first studies to investigate the use of Astym treatment on scar tissue following treatment for breast cancer. There is very limited evidence using Astym treatment on scar tissue following mastectomy, and this study demonstrates the importance of early PT intervention.

A limitation of the study was that some of the BCSs referred for Astym treatments were 8 months post-op mastectomy. These BCSs still demonstrated improvement, but the majority were referred earlier in their journey to recovery. Future studies should investigate Astym treatment at a set time following surgery of 4 weeks, for example, to support early intervention further. Also, a purpose of the study was to initially determine the effects of Astym treatment on women in general following mastectomy. Astym therapy includes elements of therapeutic loading, and patients also received MLD and postural reeducation. Future studies should assess the effects of each of the interventions alone and include a control group.

The implications of this study are that clinicians need to recognize that the scar tissue which develops postoperatively following a mastectomy or treatments for breast cancer needs to be addressed early to prevent shoulder dysfunction and significant quality-of-life issues. ${ }^{2}$ Astym therapy is an appropriate intervention that therapists should consider to improve functional outcomes.

\section{Conclusion}

The results of this study indicate that Astym therapy may be an effective treatment option to address the complications of scar tissue and soft tissue dysfunction that often result from breast cancer treatment but go untreated. Astym therapy improved shoulder range of motion and functional ability following a mastectomy. Further studies are necessary to confirm the findings in randomized controlled trials.

\section{Acknowledgments}

The authors would like to acknowledge the Baptist Health Lexington Medical Library staff who helped with this project. A small grant was provided by Performance Dynamics, Inc., to Baptist Health Lexington to offset some of the direct costs it incurred by allowing the study to take place at this institution.

\section{Disclosure}

Dr Davies is an occasional clinical consultant for Performance Dynamics, Inc., for Astym therapy advice in postmastectomy patients. The authors report no other conflicts of interest in this work.

\section{References}

1. American Cancer Society. Cancer Facts and Figures 2013-14. Atlanta, GA: American Cancer Society, Inc; 2013.

2. Smoot B, Wampler M, Topp KS. Breast cancer treatments and complications: implications for rehabilitation. Rehabil Oncol. 2009;27(3):16-26.

3. National Cancer Institute. Surveillance Epidemiology and End Results (SEER) stat fact sheet. Breast cancer. Available from: http://seer.cancer. gov/csr/1975_2006/results_single/sect_01_table.01.pdf. Accessed June $10,2010$.

4. Cheville AL, Troxel AB, Basford JR, Koprnblith AB. Prevalence and treatment patterns of physical impairments in patients with metastatic breast cancer. J Clin Oncol. 2008;26:2621-2629.

5. Leidenius M, Leppanen E, Krogerus L, von Smitten K. Motion restriction and axillary web syndrome after sentinel node biopsy and axillary clearance in breast cancer. Am J Surg. 2003;185(2):127-130.

6. Sevier TL, Stegink-Jansen CW. Astym treatment vs. eccentric exercise for lateral elbow tendinopathy: a randomized controlled clinical trial. PeerJ. 2015:3:e967. doi:10.7717/peerj.967.

7. Davidson CJ, Ganion L, Gehlsen GM, Verhoestra B, Roepke JE, Sevier TL. Rat tendon morphologic and functional changes resulting from soft tissue mobilization. Med Sci Sports Exerc. 1997;29(3):313-319.

8. Gehlsen GM, Ganion LR, Helfst RH. Fibroblast responses to variation in soft tissue mobilization pressure. Med Sci Sports Exerc. 1999;31(4):531-535.

9. Henry P, Panwitz B, Wilson JK. Treatment of a bilateral total knee replacement using augmented soft tissue mobilization. Phys Ther Case Rep. 1999;2(1):27-30.

10. Henry P, Panwitz B, Wilson JK. Rehabilitation of a post-surgical patella fracture: a case study. Physiotherapy. 2000;86(3):139-142. 
11. Slaven EJ, Mathers J. Management of chronic ankle pain using joint mobilization and Astym ${ }^{\circledR}$ treatment: a case report. J Man Manip Ther. 2011;19(2):108-112.

12. Branford OA, Brown RA, McGrouther DA, Grobbelaar AO, Mudera V. Shear-aggregated fibronectin with anti-adhesive properties. J Tissue Eng Regen Med. 2011;5(1):20-31. doi:10.1002/term.284.

13. McDonald JA, Kelley DG, Broekelmann TJ. Role of fibronectin in collagen deposition: Fab' to the gelatin-binding domain of fibronectin inhibits both fibronectin and collagen organization in fibroblast extracellular matrix. J Cell Biol. 1982;92(2):485-492.

14. Davies CC, Brockopp D. Use of ASTYM treatment on scar tissue following surgical treatment for breast cancer: a pilot study. Rehabil Oncol. 2010;28(32):3-12.

15. Daniels CJ, Morrell AP. Chiropractic management of pediatric plantar fasciitis: a case report. J Chiropr Med. 2012;11(1):58-63.

16. Solecki TJ, Herbst EM. Chiropractic management of a postoperative complete anterior cruciate ligament rupture using a multimodal approach: a case report. J Chiropr Med. 2011;10(1):47-53.

17. Schaefer JL, Sandrey MA. Effects of a 4-week dynamic-balancetraining program supplemented with graston instrument-assisted soft-tissue mobilization for chronic ankle instability. J Sport Rehabil. 2012;21(4):313-326.

18. Blanchette MA, Normand MC. Augmented soft tissue mobilization vs natural history in the treatment of lateral epicondylitis: a pilot study. J Manipulative Physiol Ther. 2011;34(2):123-130.

19. Vardiman JP, Siedlik J, Herda T, et al. Instrument-assisted soft tissue mobilization: effects on the properties of human plantar flexors. Int $J$ Sports Med. 2015;36(3):197-203.

20. McConnell S, Beaton DE, Bombardier C. The DASH Outcome Measure User's Manual. Toronto, Canada: Institute for Work and Health; 1999.

21. Swisher AK, Davison C, Aranda R, et al. Frequency and severity of self-reported upper extremity impairments, activity limitation and participation restrictions following breast cancer treatment. Rehabil Oncol. 2010;28(1):3-9.
22. Hudak P, Amadio P, Bombardier C. Development of an upper extremity outcome measure: the DASH (Disabilities of the Arm, Shoulder and Hand). Am J Ind Med. 1966;29:602-608.

23. Stratford P, Guill C, Westaway M, Brinkley J. Assessing disability and change on individual patients: a report of a patient specific measure. Physiother Can. 1995;47:258-263.

24. Tengrup I, Tennvall-Nittby L, Christiansson I, Laurin M. Arm morbidity after breast-conserving therapy for breast cancer. Acta Oncol. 2000;39(3):393-397.

25. Gosselink R, Rouffaer L, Vanhelden P, Piot W, Trooster TCM. Recovery of the upper limb function after axillary dissection. J Surg Oncol. 2003;83:204-211.

26. Lotze M, Duncan M, Gerber L, Woltering E, Rosenberg S. Early versus delayed shoulder motion following axillary dissection. Ann Surg. 1981;193(3):288-295.

27. Blomqvist L, Stark B, Engler N, Malm M. Evaluation of arm and shoulder mobility and strength after modified radical mastectomy and radiotherapy. Acta Oncol. 2004;43(3):280-283.

28. Haynes K, Walton JR, Szomor ZL, Murrell GAC. Reliability of five methods for assessing shoulder range of motion. Aust J Physiother. 2001;47(4):289-294.

29. Atroshi I, Gummesson C, Andersson B, Dahlgren E, Johansson A. The Disabilities of the Arm, Shoulder and Hand (DASH) outcome questionnaire. Reliability and validity of the Swedish version evaluated in 176 patients. Acta Orthop Scand. 2000;71(6):613-618.

30. Beaton DE, Wright JG, Katz JN. Development of the QuickDASH: comparison of three item-reduction approaches. J Bone Joint Surg. 2005;87(5):1038-1046.

31. Gummesson C, Atroshi I, Ekdahl C. The disabilities of the arm, shoulder and hand (DASH) outcome questionnaire: longitudinal construct validity and measuring self-rated health change after surgery. $B M C$ Musculoskelet Disord. 2003;4:11.

32. Muir SW, Corea CL, Beaupre L. Evaluating change in clinical status: reliability and measures of agreement for the assessment of glenohumeral range of motion. NAm J Sports Phys Ther. 2010;5(3):98-110. 


\section{Supplementary materials}

Table SI Clothing restriction following breast surgery questionnaire (please rate your ability to do the following in the last week by circling the number by the appropriate response)

\begin{tabular}{|c|c|c|c|c|c|}
\hline & No difficulty & Mild difficulty & Moderate difficulty & Severe difficulty & Unable \\
\hline Wear a bra or prosthesis & I & 2 & 3 & 4 & 5 \\
\hline Wear a bra or prosthesis all day & I & 2 & 3 & 4 & 5 \\
\hline Tolerate some fabrics against your skin in affected area & I & 2 & 3 & 4 & 5 \\
\hline Touch the scars from the mastectomy & I & 2 & 3 & 4 & 5 \\
\hline Ability for someone else to touch the mastectomy site & I & 2 & 3 & 4 & 5 \\
\hline Look at your scars from breast surgery & I & 2 & 3 & 4 & 5 \\
\hline Feel less feminine since cancer & I & 2 & 3 & 4 & 5 \\
\hline Tolerate people looking at you & I & 2 & 3 & 4 & 5 \\
\hline Comments: & & & & & \\
\hline
\end{tabular}

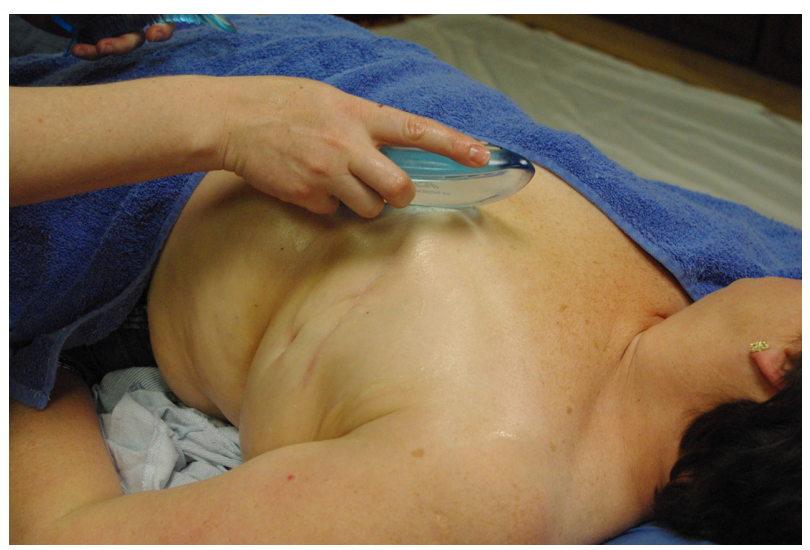

Figure SI Demonstrates the use of one of the Astym therapy tools over a mastectomy scar.

\section{Publish your work in this journal}

Breast Cancer: Targets and Therapy is an international, peerreviewed open access journal focusing on breast cancer research, identification of therapeutic targets and the optimal use of preventative and integrated treatment interventions to achieve improved outcomes, enhanced survival and quality of life for the cancer patient.
View the full aims and scopes of this journal here. The manuscript management system is completely online and includes a very quick and fair peer-review system, which is all easy to use. Visit http:// www.dovepress.com/testimonials.php to read real quotes from published authors

\footnotetext{
Submit your manuscript here: http://www.dovepress.com/breast-cancer---targets-and-therapy-journal
} 